\title{
Fungal Infection in Lung Transplantation
}

\author{
Cassie C. Kennedy, MD ${ }^{1,2}$ Kelly M. Pennington, MD ${ }^{1,2}$ Elena Beam, MD ${ }^{2,3}$ Raymund R. Razonable, MD²,3
}

${ }^{1}$ Division of Pulmonary and Critical Care Medicine, Mayo Clinic, Rochester, Minnesota

2 William J. von Liebig Center for Transplantation and Clinical

Regeneration, Mayo Clinic, Rochester, Minnesota

3 Division of Infectious Disease, Mayo Clinic, Rochester, Minnesota

Address for correspondence Cassie C. Kennedy, MD, Division of Pulmonary and Critical Care Medicine, Mayo Clinic, 200 First Street SW, Rochester, MN 55905 (e-mail: kennedy.cassie@mayo.edu).

Semin Respir Crit Care Med 2021;42:471-482.
Abstract
Keywords
- lung transplant
- fungal infection
- immuno- compromised
- antifungal prophylaxis

Invasive fungal infections threaten lung transplant outcomes with high associated morbidity and mortality. Pharmacologic prophylaxis may be key to prevent posttransplant invasive fungal infections, but cost, adverse effects, and absorption issues are barriers to effective prophylaxis. Trends in fungal infection diagnostic strategies utilize molecular diagnostic methodologies to complement traditional histopathology and culture techniques. While lung transplant recipients are susceptible to a variety of fungal pathogens, Candida spp. and Aspergillus spp. infections remain the most common. With emerging resistant organisms and multiple novel antifungal agents in the research pipeline, it is likely that treatment strategies will continue to evolve.
Fungal infections lead to significant morbidity in lung transplant recipients. In addition, they have been directly and indirectly associated with increased mortality. ${ }^{1}$ There are multiple reasons that account for the increased risk of fungal infections after lung transplantation. First, lung transplant patients receive high levels of pharmacologic immunosuppression to prevent or treat allograft rejection. This results in a functionally impaired immune system that predisposes to the occurrence of opportunistic fungal infections. Second, the lung is constantly exposed to the environment, allowing the direct access of fungal pathogens into the allograft. The hospital environment has occasionally been a source of fungal pathogens, with sporadic outbreaks of fungal infections in lung transplant units. Third, lung transplantation surgery impairs local physical and physiologic host defenses such as mucociliary clearance and cough mechanisms, respectively, impairing the transplant recipients' mechanisms of microbial clearance. Fourth, many lung transplant candidates have structural abnormalities that predispose to colonization with microbial pathogens, including fungi. Such colonization of sinuses and lung parenchyma with fungal pathogens has been associated with increased incidence of invasive fungal infections (IFIs) after lung transplantation. ${ }^{2}$

There is ongoing work to clarify and standardize the definitions of IFI. A consensus statement published in 2019 defined breakthrough fungal infections as any IFI that occurs during an exposure to antifungal therapy, including fungi outside of the spectrum of activity of the antifungal. ${ }^{3}$ The IFI should occur within one dosing interval of the antifungal medication to be considered a breakthrough IFI. ${ }^{3}$ The group also offered definitions for persistent, refractory, and relapsed infections in this document.

\section{Epidemiology}

The 1-year cumulative incidence of fungal infections in lung transplant recipients is estimated to be $10 \%$, although the rates vary widely depending on multiple factors. ${ }^{4,5}$ The occurrence and type of fungal infection depends on the intensity of physical and functional immunosuppression, the timing since transplantation, the type of antimicrobial prophylaxis, and the presence of colonization prior to transplantation. Overall, the median time to onset is roughly 11 months after lung transplantation. ${ }^{4,5}$

The majority of IFIs in lung transplant recipients are due to Aspergillus and Candida species. Candida sp. infections generally occur early after lung transplantation, usually as a complication of hospitalization and transplant surgery, in the form of mediastinitis, pleuritis, empyema, or surgical site infection. Bloodstream infection secondary to Candida species also occurs early, usually as a complication of indwelling
Issue Theme Lung Transplantation: Controversies and Evolving Concepts; Guest Editors: John A. Belperio, MD, and Scott M. Palmer, MD (c) 2021. Thieme. All rights reserved. Thieme Medical Publishers, Inc., 333 Seventh Avenue, 18th Floor, New York, NY 10001, USA
DOI https://doi.org/ 10.1055/s-0041-1729173. ISSN 1069-3424. 
vascular catheters or urinary catheters. Catheter-associated urinary tract infection with Candida species may also occur during the initial transplant hospitalization.

The 12-month cumulative incidence of invasive mold infection is 5.5\%. Aspergillus species is the most common invasive mold infection, accounting for $73 \%$ of cases reported in a surveillance network of 15 lung transplant centers. ${ }^{4,5}$ Most of the cases of aspergillosis occur during the first 6 months, and a median of 3.2 months, after lung transplantation. The vast majority of Aspergillus infections present as pneumonia or tracheobronchitis, with a risk of systemic and multiorgan dissemination in highly immune compromised patients.

Non-Aspergillus mold infections such as agents of mucormycosis, Alternaria, Fusarium, Scedosporium, among others occur at a much lower rate, at $27 \%$, and at a much later time point since transplant surgery. ${ }^{4,5}$ Certain groups of lung transplant patients are at increased risk of infections due to endemic fungi such as Histoplasma capsulatum, Blastomyces dermatitidis, and Coccidioides immitis.

However, clinicians should be aware that geographic boundaries of endemic mycoses are changing. ${ }^{6}$ Cases of histoplasmosis have been reported to occur outside of the expected geographic endemic location, even in individuals without associated travel to an endemic area. $C$. immitis has been noted in soil environment, with local acquisition of patients in Washington State. Blastomyces percursus and Blastomyces emzantsi spp. have been better described as causes of human blastomycosis in South Africa. ${ }^{6-8}$

Geography seems to also play a role in the resistance patterns of certain IFIs. Clinicians should be aware of the potential for increased baseline azole resistance of Aspergillus sp. reported in Europe, particularly the Netherlands where 11 to $18 \%$ of isolates may be resistant. ${ }^{9}$ Half of the environmental samples of Aspergillus fumigatus in azole-fungicide-containing plant waste in the Netherlands were azole resistant and carried resistance genes. ${ }^{10}$ However, azole resistance has not been common in the United States. Only 1 of 181 A. fumigatus clinical isolates in the United States had azole resistance. ${ }^{11}$

\section{Prevention}

Prevention of mold infections typically begins with avoidance of high-risk exposures (e.g., avoiding compost and areas of construction and demolition) and utilizing respiratory protection whenever necessary. The use of antimicrobial prophylaxis is the most common approach-whether this is universal or a targeted approach. Universal antimicrobial prophylaxis to prevent Pneumocystis jirovecii is standard of care in lung transplant recipients, typically with sulfamethoxazole-trimethoprim. ${ }^{12}$ The use of antifungal prophylaxis against mold infection is increasingly utilized following lung transplantation, although the details of this practice are highly variable. Surveys to define clinical practice regarding antifungal prevention strategies in lung transplant recipients indicate a trend toward increased utilization of antifungal prophylaxis protocols over time. Although approximately 59\% of lung transplant programs utilized a universal prophylaxis strategy in a worldwide survey published in $2011,{ }^{13}$ by 2019 the rate was $90 \%$ in a survey of U.S. lung transplant centers. ${ }^{14}$

Antifungal prophylaxis against mold infection is commonly employed in the form of one of three strategies: (1) use of a topical inhaled antifungal agent (e.g., Amphotericin B nebulizer) to prevent postoperative tracheobronchitis; (2) use of a systemic antimold medication to prevent IFIs; or (3) use of both types of agents. ${ }^{14,15}$

The antimicrobial agents most commonly used for systemic antifungal prophylaxis are voriconazole and itraconazole. Barriers to prophylaxis are side effects, cost, and malabsorption. ${ }^{16}$ A large retrospective study $(N=662)$ utilizing OptumLabs Data Warehouse claims data demonstrated that approximately 60\% of lung transplant recipients filled antifungal prophylaxis following the index transplant hospitalization between 2005 and 2018. ${ }^{17}$ This study was the first to demonstrate a significant survival benefit for lung transplant recipients obtaining prophylaxis in the first year following lung transplantation, compared with propensity-weighted controls who did not (8.4 events per 100 person-years compared with 19.5 in those without, $p=0.003) .{ }^{17}$ The duration of antifungal prophylaxis, utility of pretransplant respiratory culture data (to assess fungal colonization), and specific medication regimen remains controversial. Although some centers use antifungal prophylaxis in all lung transplant recipients (the so-called universal prophylaxis), other centers limit antifungal prophylaxis to those with high-risk diagnoses or to those patients with evidence of colonization by fungal organisms on pre- or posttransplant respiratory cultures. ${ }^{18,19}$

\section{Fungal Diagnostic Tools}

Definitive diagnosis of IFI after lung transplantation relies on obtaining tissue for direct, histopathologic visualization of tissue invasion or fungal organisms in culture of specimens from sterile sites. Bronchoscopic and computed tomography (CT)-guided percutaneous lung biopsies are the two major ways to obtain tissue for histopathology and cultures for suspected pulmonary fungal disease. Fungal cultures and antifungal susceptibility tests generally guide the definitive management.

Molecular methods directed at detecting fungal antigens and deoxyribonucleic acid (DNA) allow for more rapid diagnosis when applied and interpreted in the appropriate clinical context. A recent study analyzed samples where hyphae were present on histopathology from immunosuppressed patients, and reported that broad-range fungal polymerase chain reaction (PCR) had sensitivity of $90 \%$ and a specificity of $86 \%{ }^{20}$ Pathogen-specific Aspergillus PCR on hyphae-positive tissue had a sensitivity of $89 \%$, but specificity was only $58 \%{ }^{20}$ - Table 1 lists the characteristics, limitations, and controversies related to modern diagnostic tools. In an immunosuppressed population, antigen and DNA detection is favored over antibody testing, as antibody response can be unpredictable.

Galactomannan: Detection of galactomannan using enzyme immunoassay (EIA) has been utilized for nearly two decades. ${ }^{21}$ Galactomannan is a major cell wall constituent of Aspergillus spp. and is released during fungal replication. ${ }^{22}$ 
Table 1 Serologic tests for detection of fungal antigens and DNA for common fungal pathogens in lung transplant recipients

\begin{tabular}{|c|c|c|}
\hline Diagnostic test & Clinical use & Caveats \\
\hline Galactomannan & $\begin{array}{l}\text { Serum: } \\
\text { - Diagnose invasive Aspergillus spp. or related } \\
\text { molds in neutropenic patients } \\
\text { BAL: } \\
\text { - Diagnose pulmonary Aspergillus spp. or related } \\
\text { molds in neutropenic and nonneutropenic } \\
\text { patients }\end{array}$ & $\begin{array}{l}\text { - Serum galactomannan has low sensitivity in } \\
\text { nonneutropenic patients } \\
\text { - Can cross-react with other mold species; culture } \\
\text { data are still necessary } \\
\text { - False positive can occur from dietary ingestion, } \\
\text { penicillin antibiotics } \\
\text { - Effect of antifungal medications on sensitivity is } \\
\text { unknown }\end{array}$ \\
\hline$\beta-1,3-D$ glucan & $\begin{array}{l}\text { - Used in conjunction with other diagnostic tools to } \\
\text { screen for invasive fungal infection } \\
\text { - May allow for safe discontinuation of antifungal } \\
\text { medications in high-risk populations }\end{array}$ & $\begin{array}{l}\text { - Cryptococcus spp. and Mucorales will not cause } \\
\text { elevation } \\
\text { - Should not be used as a standalone diagnostic test } \\
\text { for invasive fungal disease } \\
\text { - False positive can occur in patient receiving blood } \\
\text { products, those on hemodialysis or cardiopul- } \\
\text { monary bypass } \\
\text { - Effect of antifungal medications on sensitivity is } \\
\text { unknown }\end{array}$ \\
\hline Aspergillus PCR & $\begin{array}{l}\text { Serum: } \\
\text { - Single test can help rule out invasive Aspergillus } \\
\text { - Two positive serum tests can help rule in invasive } \\
\text { Aspergillus } \\
\text { BAL: } \\
\text { - Can identify the presence of Aspergillus spp. }\end{array}$ & $\begin{array}{l}\text { - Efforts to standardize PCR methods are ongoing } \\
\text { - Most studies are in hematologic malignancy } \\
\text { population } \\
\text { - BAL cannot distinguish between colonization and } \\
\text { infection } \\
\text { - Antifungal medications significantly reduce } \\
\text { sensitivity }\end{array}$ \\
\hline Mucorales PCR & - Not clinically available & $\begin{array}{l}\text { - Antifungal medications significantly reduce } \\
\text { sensitivity }\end{array}$ \\
\hline $\begin{array}{l}\text { T2Candida } \\
\text { nanodiagnostic } \\
\text { panel }\end{array}$ & $\begin{array}{l}\text { Whole blood: } \\
\text { - Rapid detection of candidemia in high-risk } \\
\text { populations }\end{array}$ & $\begin{array}{l}\text { - Not evaluated for detection of deep-seated } \\
\text { infection in the absence of candidemia } \\
\text { - Effect of antifungal medications on sensitivity is } \\
\text { unknown }\end{array}$ \\
\hline
\end{tabular}

Abbreviations: BAL, bronchoalveolar lavage; DNA, deoxyribonucleic acid; PCR, polymerase chain reaction.

Serum galactomannan has the greatest sensitivity for the diagnosis of IFI in patients with severe neutropenia $(<500$ neutrophils per $\mathrm{mm}^{3}$ ). In nonneutropenic patients, however, circulating galactomannan is consumed by the intact immune system. As such, the sensitivity of serum galactomannan for the diagnosis of IFI is estimated to be $71 \%$ in patients with hematologic malignancy and hematopoietic stem cell transplant and only $25 \%$ in nonneutropenic solidorgan transplant patients. ${ }^{23}$ Galactomannan EIA sensitivity is improved to $88 \%$ in solid-organ transplant patients when utilizing bronchoalveolar lavage (BAL) specimens rather than serum or plasma. ${ }^{23}$ The sensitivity of BAL galactomannan is reported between 57 and $100 \%$ in all-comers. ${ }^{24-29}$ The specificity of galactomannan is estimated to be around $90 \%$ for serum and BAL specimens. ${ }^{23-29}$

Galactomannan: EIA can cross-react with mold species that are closely related to Aspergillus spp. such as Fusarium spp. and Penicillium spp. ${ }^{30}$ In the case of pathologic molds such as Fusarium spp., this can be a helpful diagnostic adjunct. In the case of nonpathogenic Penicillium spp., this cross-reaction can lead to false-positive results with Penicillium spp. colonization or exposure to penicillin antibiotics ${ }^{31}$; however, the purification process for penicillin antibiotics has improved in recent years; so, false positives for this reason may no longer be a concern. Dietary ingestion of galactomannan in patients with mucositis may also be a cause for false-positive serum galactomannan. The impact of dietary galactomannan ingestion on BAL galactomannan is unknown. In the modern era where antifungal prophylaxis is commonplace for lung transplant recipients, it is important to note that the impact of cell-wall targeted antifungal medications, such as triazoles, on the sensitivity of serum or BAL galactomannan assays is unknown.

$\beta-1,3-D$ glucan: Quantitative detection of $\beta-1,3-D$ glucan has been utilized to aid in the diagnosis of IFI since the 1990s. ${ }^{32} \beta-1,3-D$ glucan is contained in the cell wall of most fungal organisms, with the notable exceptions of Blastomyces sp., Cryptococcus spp., and Mucorales. While $\beta-1,3-D$ glucan is an abundant constituent of Candida spp. cell-wall and elevated assays have traditionally been associated with invasive candidiasis, $\beta-1,3-D$ glucan assays do not differentiate Candida spp. infection from other fungi. The sensitivity and specificity of $\beta-1,3-D$ glucan for invasive candidiasis is around 80 and $60 \%$, respectively. ${ }^{33}$ An elevated $\beta-1,3-D$ glucan level must be considered in appropriate clinical context. While low $\beta-1,3-D$ glucan levels in high-risk populations suspected of invasive candidiasis may allow for the safe discontinuation of antifungal medications, ${ }^{34,35}$ no evidence exists to support the initiation of antifungal treatment based solely on elevated $(>80 \mathrm{pg} / \mathrm{mL}$ ) $\beta-1,3-D$ glucan levels. ${ }^{33}$ 
$\beta-1,3-D$ glucan can be falsely elevated in patients receiving blood transfusions or intravenous immunoglobulin, and those on hemodialysis or cardiopulmonary bypass. ${ }^{36}$ Notably, $\beta-1,3-D$ glucan levels are characteristically high $(>300 \mathrm{pg} / \mathrm{mL})$ among patients with Pneumocystis pneumonia. ${ }^{37}$ Similar to galactomannan, the effect of cell-wall-targeted antifungal prophylaxis or therapy on assay sensitivity is unknown.

Aspergillus PCR: While efforts are ongoing to standardize Aspergillus PCR methods, no universally accepted method for the detection of Aspergillus spp. currently exists. Methods vary by DNA extraction protocol, amplification technique, positivity threshold, and specimen type. A meta-analysis of serum Aspergillus PCR-based studies found a sensitivity of $80 \%$ and specificity of $79 \%$ for a single positive test, and sensitivity of $58 \%$ and specificity of $96 \%$ for two positive tests. ${ }^{38}$ This indicates that single serum PCR may be helpful to rule out disease and two positive serum PCR tests may be helpful to rule in disease. However, many of the studies on Aspergillus PCR are on the detection or early diagnosis of invasive aspergillosis in patients with hematologic malignancy; so, applicability to lung transplant populations is unknown. Additionally, antifungal prophylaxis or therapy appears to reduce the positive predictive value of serum Aspergillus PCR from around $63 \%$ to a mere $5 \%,{ }^{39}$ indicating that the test may be best applied to antifungal naive patients.

Aspergillus: PCR can be used on BAL specimens. While the sensitivity for BAL Aspergillus PCR is greater than $90 \%,{ }^{40}$ it cannot distinguish between colonization and infection. Secondary to different BAL and PCR techniques, a threshold level to separate colonization and infection has not been established. A negative PCR on BAL may be helpful to exclude invasive aspergillosis, ${ }^{27}$ but similar to serum PCR antifungal medications prior to testing greatly reduce the sensitivity. ${ }^{40}$

T2Candida nanodiagnostic panel: T2Candida panel is the only FDA-approved test to detect Candida spp. on whole blood samples. Using a combination of nuclear magnetic resonance and PCR, the platform allows for rapid ( $<3$ hours) detection of C. albicans, C. tropicalis, C. parapsilosis, C. krusei, and $C$. glabrata. ${ }^{41,42} \mathrm{~A}$ multicenter trial of hospitalized patients with candidemia found the sensitivity of the T2Candida panel to be $89 \%$ with a positive predictive value of $84 \%$ in a setting of $10 \%$ candidemia prevalence. ${ }^{43}$ The clinical utility of the T2Candida panel for suspected candidemia has not been fully evaluated. In patients at risk for candidemia, empiric antifungal therapy may be more clinically impactful than using the T2Candida panel. ${ }^{44}$ The T2Candida panel has not been evaluated for the detection of deep-seated Candida spp. infections.

Endemic fungi antigen and antibody detection: B. dermatitidis, H. capsulatum, and Coccidioides spp. are the endemic fungi that cause disease in the United States. Aside from culture and direct visualization, diagnostic tests include antibody testing and antigen detection on urine, BAL, and serum samples.

B. dermatitidis and $H$. capsulatum: antigen testing detects capsule proteins. Secondary to their similarities in the capsule protein structure, there is a significant degree of crossreactivity between these two fungi, lowering the specificity of these tests. Urine Blastomyces antigen testing has an overall sensitivity of 76 to $93 \%$ and a specificity of $80 \%{ }^{45-47}$ Serum and BAL Blastomyces antigen testings have sensitivities less than $70 \%{ }^{47}$ Conversely, Histoplasma serum and urine antigen testings have similar sensitivities (around $80 \%$ for disseminated disease and $65 \%$ for pulmonary disease) ${ }^{48,49}$ Antigen testing is not routinely used to diagnose Coccidioides spp. infection.

The role for antibody testing in diagnosing active B. dermatitidis and H. capsulatum is limited. Traditional antibody testing for Blastomyces targets antibodies to a surface $A$ antigen and has a sensitivity of $77 \%$ when using EIA techniques. ${ }^{50}$ EIA techniques targeting antibodies to the surface protein, BAD-1, improved sensitivity to $88 \%{ }^{51}$ but is not commercially available. Compared with immunodiffusion and complement fixation, EIA techniques likewise appear to have the best sensitivity and specificity for diagnosing $H$. capsulatum infection. The sensitivity and specificity is 89 and $92 \%$, respectively, when combining immunoglobulin $\mathrm{M}$ and immunoglobulin $\mathrm{G}$ antibodies. ${ }^{49}$ Like other antibody methods, the host immune response influences the sensitivity of the test. The sensitivity is likely lower in lung transplant patients.

Antibody testing is the most commonly used method for diagnosing Coccidioides spp. infection. EIA has high sensitivity of $90 \%$, and it is used to screen for disease. ${ }^{52}$ Immunodiffusion and complement fixation techniques are used for confirmatory testing. As with other antibody tests, the sensitivity is likely lower in lung transplant patients, potentially due to the impaired antibody production in this population.

\section{Important Pathogens}

Fungal infection in lung transplant recipient can manifest as tracheobronchitis, fungal pneumonia, mediastinal infection, or disseminated fungal infection. Common fungal pathogens in lung transplant recipients and resultant disease manifestations are reviewed below.

\section{Candida}

Candida spp. can cause a spectrum of disease. Invasive candidiasis is the presence of Candida spp. in the blood stream, deep-seated tissue, or both. Candidemia is one of the most common nosocomial blood stream infections in critically ill patients and is associated with high mortality. ${ }^{53}$ Invasive Candida spp. infections occur most commonly within the first 3 months posttransplant and are often the result of indwelling vascular catheters, surgical site infections, or airway anastomotic dehiscence resulting in pleural space contamination. ${ }^{53}$ Risk factors for invasive candidiasis in lung transplant recipients include prolonged critical illness, neutropenia, and prolonged antibacterial therapy. The most common presentation in lung transplant recipients is line-related candidemia, candida empyema, or surgical site infection. Source control and prompt initiation of antifungal therapy (-Table 2 ) are associated with improved survival. ${ }^{54}$ 
Table 2 General treatment choices of selected fungal infections in lung transplant recipients

\begin{tabular}{|c|c|}
\hline Fungal infection & Recommended antifungal treatment \\
\hline Aspergillus sp. & $\begin{array}{l}\text { Invasive aspergillosis } \\
\text { - Voriconazole is treatment of choice } \\
\text { Alternative therapies: } \\
\text { - Liposomal amphotericin B product; inhalation may be given for tracheobronchitis } \\
\text { in addition to systemic therapy } \\
\text { - Other triazoles: posaconazole, isavuconazole } \\
\text { - Echinocandins: caspofungin, anidulafungin, micafungin } \\
\text { - Combination therapy may be considered }\end{array}$ \\
\hline Candida sp. & $\begin{array}{l}\text { Candidemia and invasive candidiasis } \\
\text { - Echinocandins as initial empiric therapy: anidulafungin, micafungin, caspofungin } \\
\text { Alternatives: } \\
\text { - Fluconazole as alternative empiric therapy if azole-resistance is not a concern } \\
\text { - Liposomal amphotericin B } \\
\text { Targeted therapy based on susceptibility testing }\end{array}$ \\
\hline $\begin{array}{l}\text { Cryptococcus } \\
\text { neoformans }\end{array}$ & $\begin{array}{l}\text { Cryptococcal meningitis } \\
\text { - Induction therapy: liposomal amphotericin B with flucytosine for } 2-4 \text { wk } \\
\text { - Consolidation phase: fluconazole } 400-800 \mathrm{mg} \text { daily for } 8 \text { wk } \\
\text { - Maintenance phase: fluconazole } 200-400 \mathrm{mg} \text { daily for at least } 1 \mathrm{y} \\
\text { Extra-central nervous system cryptococcal infections } \\
\text { - Severe disease: similar treatment as meningitis } \\
\text { - Nonsevere disease: fluconazole }\end{array}$ \\
\hline Fusarium & $\begin{array}{l}\text { Invasive fusariosis } \\
\text { - Optimal antifungal therapy unclear } \\
\text { - Choices: amphotericin B, voriconazole, posaconazole, isavuconazole }\end{array}$ \\
\hline Mucormycosis & $\begin{array}{l}\text { Invasive mucormycosis } \\
\text { - Liposomal amphotericin } \mathrm{B} \text {, high dose } \\
\text { - Alternatives: posaconazole and isavuconazole }\end{array}$ \\
\hline Pneumocystis jirovecii & $\begin{array}{l}\text { Pneumocystis pneumonia } \\
\text { - Trimethoprim-sulfamethoxazole; role of steroids not proven }\end{array}$ \\
\hline $\begin{array}{l}\text { Scedosporium } \\
\text { and Lomentospora }\end{array}$ & $\begin{array}{l}\text { Invasive scedosporiosis apiospermum } \\
\text { - Voriconazole is the treatment of choice } \\
\text { Invasive lomentosporiosis prolificans } \\
\text { - Combination therapy (e.g., voriconazole plus terbinafine, others); multidrug resistant pathogen }\end{array}$ \\
\hline
\end{tabular}

\section{Molds}

\section{Aspergillus}

Aspergillus spp. are ubiquitous in the environment. The spectrum of clinical syndromes caused by Aspergillus spp. is largely dependent on host risk factors and includes respiratory tract colonization, mycetoma, and invasive aspergillosis. Invasive aspergillosis is the most serious and occurs in patients with significant immune compromise, critical illness, or underlying structural lung disease. ${ }^{55}$ Severe neutropenia and lung transplantation are the greatest risk factors for the development of invasive aspergillosis. Lung transplant recipients are at greatest risk within the first 12 months following transplantation.

Aspergilloma tends to occur in the setting of structural lung disease and presents with a cavitary lung lesion with internal debris and surrounding ground-glass opacities or consolidation on CT of the chest. The surrounding areas of ground glass and consolidation represent local invasion into the surrounding lung tissue. - Fig. 1 demonstrates a right upper lobe aspergilloma that developed in a 65-year-old double-lung transplant recipient with chronic lung allograft dysfunction-bronchiolitis obliterans phenotype. He was 8 years posttransplant and treated with pulse dose steroids for potential acute cellular rejection episode 3 months prior. BAL cultures grew A. fumigatus, and BAL galactomannan was significantly elevated. Despite targeted antifungal therapy, this patient required a right upper lobectomy, as is often required for the management of mycetoma.

Aspergillosis can have disseminated involvement in lung transplant patients with higher-intensity T-cell-targeted immunosuppression. A 63-year-old patient presented 9 months after double-lung transplant with lower extremity ulcers and declining pulmonary function. She was treated with plasma exchange, steroid bolus, and antithymocyte globulin for antibody-mediated rejection in the months prior to presentation. - Fig. 2 demonstrates a diffuse nodular infiltrate with areas of ground-glass opacity and consolidation. BAL cultures grew A. fumigatus. Transbronchial biopsy and skin biopsy demonstrated fungal elements consistent with Aspergillus spp. The patient was treated with voriconazole and reduction in immune suppression but had continued disease progression. This required salvage combination therapy with posaconazole and caspofungin. 


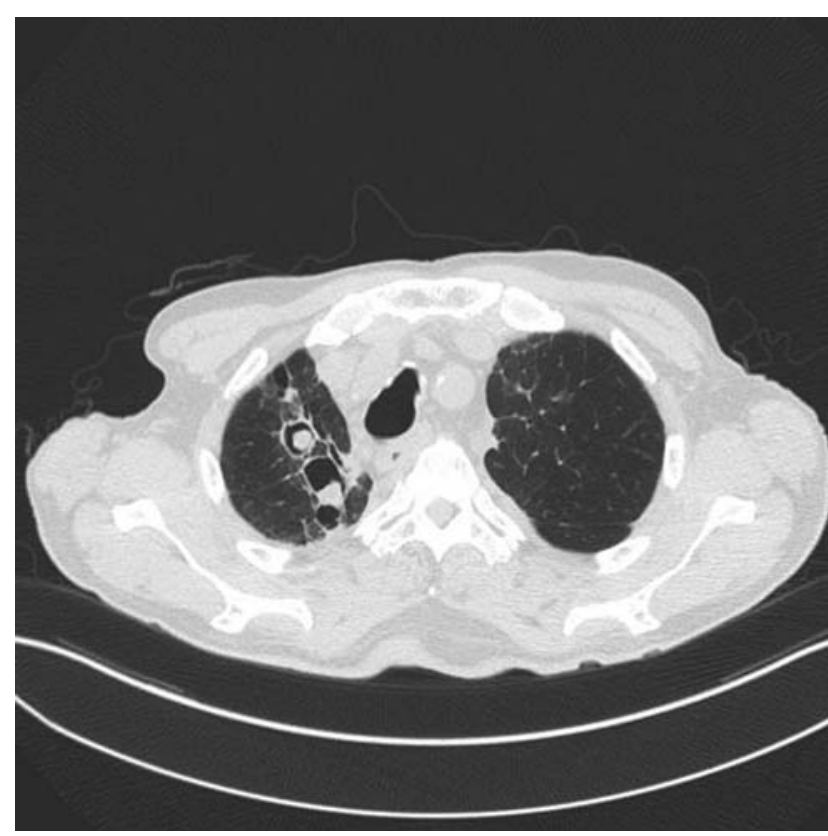

Fig. 1 Computed tomography of the chest demonstrating a right upper lobe cavity with internal debris and surrounding ground glass and consolidation consistent with a mycetoma.

\section{Fusarium}

Fusarium spp. are filamentous fungi that are found ubiquitously worldwide. They are an uncommon but important pathogen in lung transplant recipients, and are associated with high mortality. Fusarium causes infection in lung transplant recipients through inhalation and cutaneous and mucosal invasion. The respiratory system has been the portal of entry for the majority of Fusarium infections in lung transplant recipients, leading to sinusitis and pneumonia, while local cutaneous and mucosal invasion may lead to keratitis and skin and soft-tissue infections. The potential for hematogenous spread with subsequent multiorgan involvement is facilitated by severe and prolonged neutropenia, and severe T-cell immunodeficiency. ${ }^{56}$

Fusarium can cause a wide range of clinical manifestations, from superficial, locally invasive, and disseminated infections. The lungs are involved in the majority of cases of disseminated fusariosis. Radiographic studies show pneumonia, nodules, cavitary lesions, and parenchymal infiltrates. Patients usually present with cough, chest pain, and shortness of breath. Diagnosis is confirmed by the isolation of the fungi in culture. Biopsy may be performed to document invasive infection. Blood cultures may be positive. Disseminated fusariosis has poor prognosis. There is a high mortality rate, as high as $65 \%$, particularly for disseminated infections, and those with severe neutropenia and markedly impaired immune status. ${ }^{56}$

\section{Mucormycosis}

Among IFIs with highest morbidity and mortality in the lung transplant population are those in the order of Mucorales. Mucormycosis is the third most common cause of IFI among lung transplant recipients with an incidence of $2 \% 1$ year after transplant. ${ }^{4}$ The genera most commonly implicated in human infections are Rhizopus, Mucor, and Rhizomucor. ${ }^{57}$ These are classically described as broad, irregularly branched, pauci-septate ribbon-like hyphae on hematoxylin and eosin staining, and this distinct appearance allows for preliminary identification from histopathologic specimens to guide therapeutic management. Though not classical or exclusive to this infection, radiologic studies may show the reverse halo sign, describing a focal area of ground-glass attenuation surrounded by rim of consolidation. ${ }^{58}$ Rapidly developing cavitary lesions on imaging (chest radiograph [CXR] or CT scan) can be seen (-Fig. 3 ).

Mucormycosis most commonly presents as pulmonary disease, although rhinocerebral, disseminated, anastomotic, gastrointestinal, and cutaneous manifestations have all been described. ${ }^{59}$ The diagnosis is challenging, as noninvasive studies such as galactomannan and $\beta-1,3-D$-glucan and fungal blood cultures are classically negative for these organisms even in the presence of invasive disseminated disease. The possibility of mucormycosis should be considered in clinical scenarios suspicion of fungal infections especially when the host is receiving antifungal prophylaxis that is not active against this group of organisms (such as prophylaxis with fluconazole, itraconazole, or voriconazole). Transplant centers with recent cases of IFI due to mucormycosis should be aware of the implications of hospital-acquired infection and investigate such cases for causes to prevent further cases. ${ }^{60-62}$

Management relies on rapid diagnosis, aggressive and prompt surgical resection, and initiation of active antifungal therapy ( - Table 2 ). The diffuse nature of pulmonary disease due to mucormycosis that is seen after lung transplantation may limit the options for surgical resection. ${ }^{59}$ Poor outcomes in lung transplant recipients due to mucormycosis are attributed to rapid vascular invasion, necrosis, and destruction of tissues. Mortality due to mucormycosis is upward of $96 \%$ when disseminated but lower in localized pulmonary disease $(76 \%)^{57}$

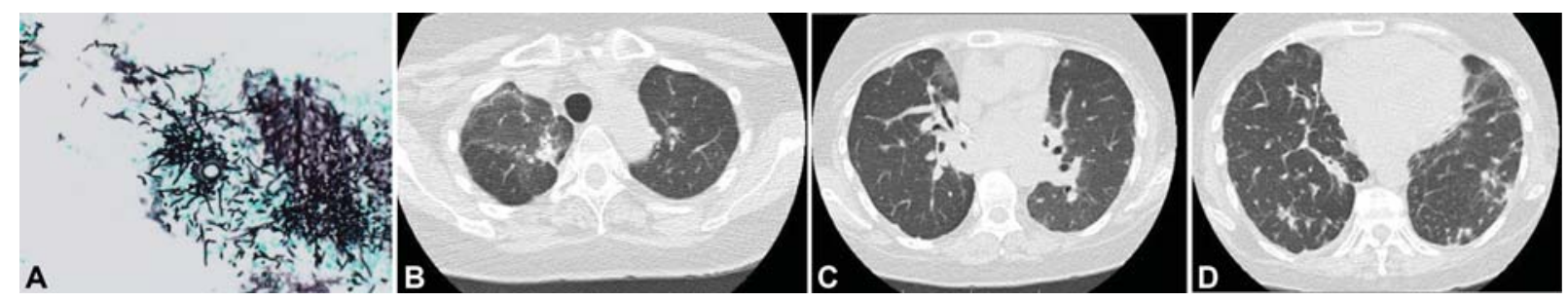

Fig. 2 Computed tomography (CT) of the chest demonstrating diffuse nodular and ground-glass infiltrates. In isolation, these CT findings are nonspecific but concerning for an evolving atypical infection. Transbronchial biopsy confirmed invasive aspergillosis. Histopathology showed acute branching hyphae with septae consistent with Aspergillus spp. Image courtesy: Dr. Audrey N. Schuetz, MD, Mayo Clinic, Rochester. 

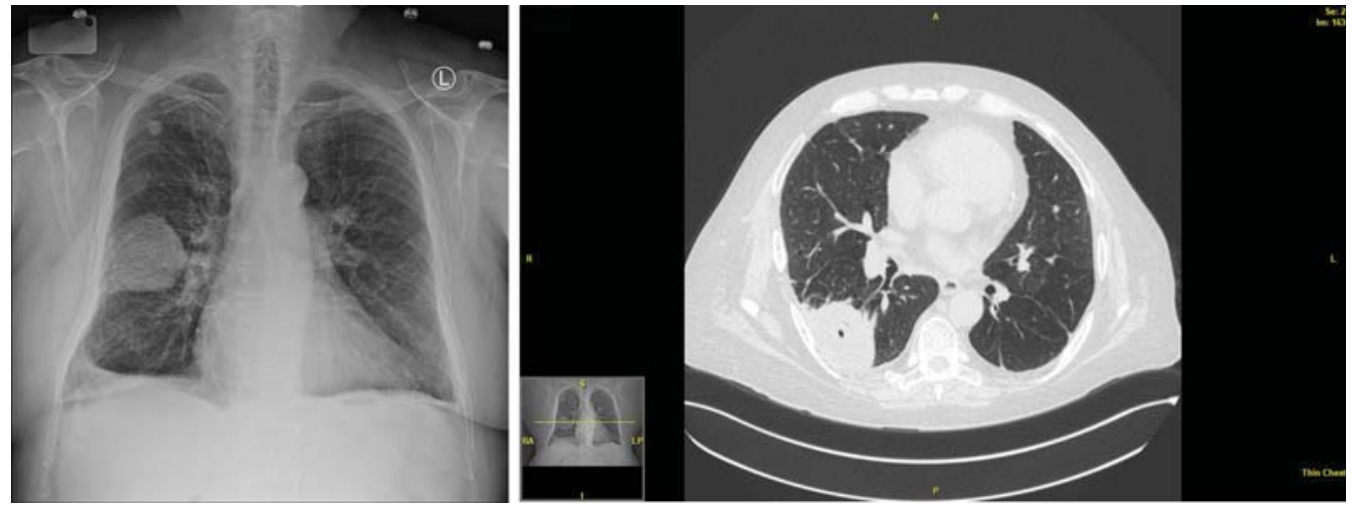

Fig. 3 (A) Chest X-ray demonstrating a cavitary mass in the right lower lobe, biopsy proven to be an infection due to Rhizopus sp. (B) Partially cavitary mass in right lower lobe with adjacent pleural thickening, proven by biopsy to be Rhizopus sp.

\section{Scedosporium}

Lung transplant recipients are at increased risk for infection with Scedosporium, particularly those with underlying cystic fibrosis. Identification of Scedosporium in BAL specimens of lung transplant recipients without evidence of infection and identification in patients with either fungal pneumonia or those with evidence of disseminated disease demonstrate the spectrum of manifestations possible with these organisms. Both hyphae and conidia can be present on histopathology specimens of infected tissue using methenamine silver fungal stains. Lowering immunosuppressive therapy and using voriconazole and surgical debridement (when possible) to treat the underlying infection is the most common strategy. Use of a second concomitant antifungal agent (e.g., terbinafine) is controversial (-Table $\mathbf{2}$ ).

\section{Dimorphic Fungi}

\section{Blastomycosis}

Blastomyces causes disease via direct inhalation of spores and can disseminate to a variety of organ systems with a predilection for skin, genitourinary tract, and lungs. ${ }^{33}$ Blastomycosis infection may present with a range of clinical syndromes that includes asymptomatic pulmonary nodules, necrotizing pneumonia, acute respiratory distress syndrome, prostatitis, and central nervous symptom infections. Host risk factors do not seem to play a significant role in the type of clinical syndrome that develops, and lung transplant recipients do not tend to be more susceptible to Blastomycosis infections. Blastomycosis always requires antifungal treatment. The type and duration of antifungal treatment depends on the clinical syndrome. ${ }^{63}$

-Fig. 4 illustrates the spectrum of respiratory disease caused by Blastomycosis. - Fig. 4A represents an incidentally discovered pulmonary nodule in a 24-year-old woman undergoing evaluation for liver transplantation for cryptogenic cirrhosis. Blastomycosis antibody and antigen testings were negative; however, Blastomycosis was identified on sputum culture. - Fig. 4B is the CXR of a previously healthy 34-year-old woman with 7-day history of pleuritic chest pain cough. Blastomyces antibody to A surface antigen by EIA and Histoplasma urinary antigen were positive. Sputum smear and culture confirmed the diagnosis of Blastomycosis pneumonia. She was treated with 6 weeks of itraconazole and made a full recovery. The CXR in - Fig. $\mathbf{4 C}$ is that of a previously healthy 26-year-old man who presented with fevers, cough, and 4-week history of pneumonia refractory to outpatient antibiotic management. His respiratory status rapidly deteriorated within 24 hours of admission requiring intubation and eventual venovenous extracorporeal membrane oxygenation. Blastomyces antibody to A surface antigen by EIA and Histoplasma urinary
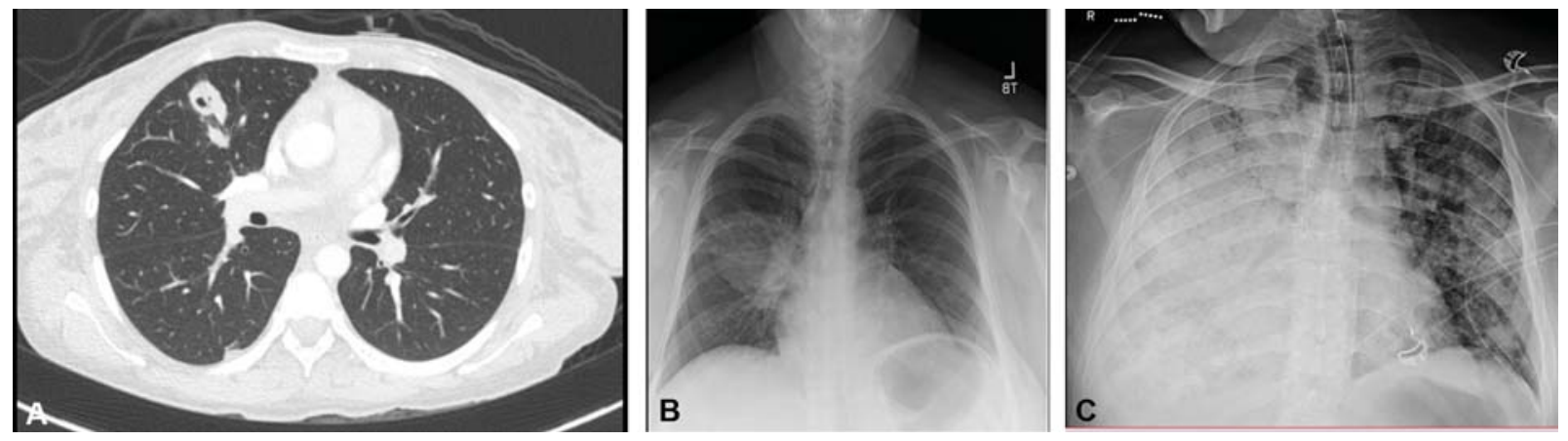

Fig. 4 (A) Computed tomography (CT) illustrating an incidentally discovered right upper lobe cavitary nodule in a 24-year-old woman undergoing evaluation for liver transplantation. Sputum culture grew Blastomycosis dermatitidis. (B) Chest roentgenogram (CXR) demonstrating right middle lobe pneumonia in a previously healthy 34-year-old woman. Diagnosis of Blastomyces pneumonia was confirmed on sputum smear and culture. (C) CXR demonstrating dense right lung consolidation and patchy left lung infiltrates in a previously healthy 26-year-old man. Blastomycosis dermatitidis was identified on bronchoalveolar lavage fungal smear. 


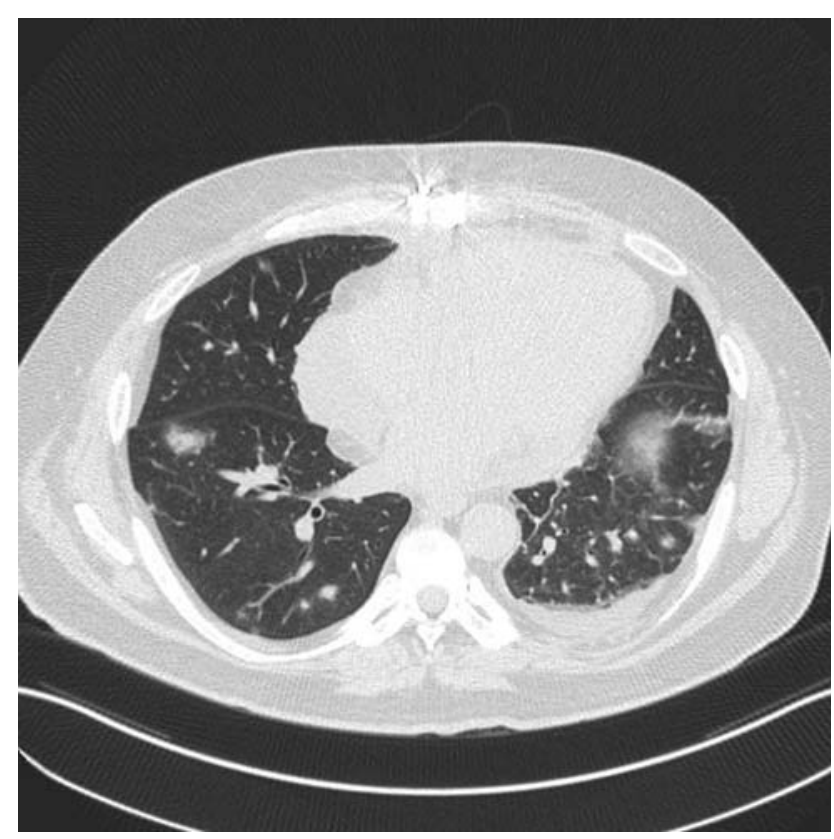

Fig. 5 Multifocal nodular opacities in lungs with surrounding groundglass halos due to coccidiomycosis 3 months after undergoing heart transplantation.

antigen were positive. BAL Blastomyces PCR, smear, and culture were also positive. After he demonstrated clearance of Blastomyces on BAL cultures, he underwent double-lung transplant for postinflammatory fibrotic lung disease. The timing of transplantation listing was decided based on culture clearance as means of identifying any remaining viable organism rather than utilizing PCR or antigen testing. Unfortunately, he succumbed to intra-abdominal complications of his prolonged critical illness.

\section{Coccidiomycosis}

C. immitis and C. posadasii are the species which cause this disease process. Disease is most commonly acquired via inhalation of spores from the environment. Endemic areas include the Southwestern United States, Mexico, and certain areas of desert regions of Central and South America. This IFI can pose numerous challenges in management, diagnosis, and prevention.

Coccidiomycosis does not always require treatment in the immunocompetent host, as it is generally a mild and selflimited infection. In immunosuppressed patients, such as those on immunosuppressive agents or with diminished cellular immunity, coccidiomycosis can present a significant challenge. Reactivation of previous latent infection can be encountered, years or even decades after the initial infection (example of posttransplant reactivation is shown in -Fig. 5). Active infection is associated with significant morbidity and mortality.

Screening of lung transplant candidates at risk for coccidiomycosis pretransplantation (via serology testing, and history and clinical questions) is important to identify those at risk and in need of antifungal prophylactic therapy. ${ }^{64}$ Similarly, lung transplant recipients residing in areas endemic for coccidiomycosis should receive primary prophylaxis after lung transplantation, for a minimum of 6 to 12 months, as well as when being treated for allograft rejection with intensification of immunosuppressive therapy. Optimal duration of prophylaxis in transplant recipients is not well established, although in some situations, such as cases of central nervous system infection, lifelong antifungal prophylaxis is generally recommended. ${ }^{64}$ Infection developing after lung transplantation most commonly presents in the first 6 to 12 months after transplant as severe pulmonary or disseminated infection. Clinicians should also be aware of uncommon but reported cases of donor-derived infection. In such situations, the lung transplant recipient should be managed with lifelong antifungal suppression. ${ }^{64,65}$

Even mild cases of infection should be investigated and treated to prevent progression to more severe or disseminated disease. Fluconazole remains the main drug for treatment and prophylaxis with the exception of severe disseminated disease, where use of lipid formulation amphotericin is recommended. ${ }^{64}$

\section{Histoplasma}

Histoplasmosis, caused by H. capsulatum, a dimorphic fungus that is endemic to the Midwestern United States, Mexico, and regions of South America with typical exposures attributed to bird or bat droppings. ${ }^{64}$ Immunocompromised lung transplant recipients can suffer an opportunistic histoplasmosis infection usually secondary to posttransplant exposure. Diagnosis of histoplasmosis infection includes respiratory samples for culture, tissue histopathology with fungal stains (-Fig. 6), and/or Histoplasma urine antigen. Severe disease warrants initiation of liposomal amphotericin; however, mild to moderate infections are typically responsive to itraconazole therapy. As such, histoplasmosis infections while taking posttransplant antifungal prophylaxis are exceedingly rare. Of note, guidelines do not endorse the need for prophylaxis for history of remote infection, although it can be considered for those with histoplasmosis within 2 years of transplant. ${ }^{64}$

\section{Pneumocystis}

Pneumocystis is a fungal organism that exclusively causes infection in immunocompromised hosts, and most commonly affects those with reduced T-cell immunity. While human immunodeficiency virus (HIV) is the most notable risk factor for the development of Pneumocystis pneumonia (PCP), PCP can occur in lung transplant recipients and has a high overall mortality rate. ${ }^{12} \mathrm{PCP}$ differs in non-HIV patient populations in that there appears to be a lower organism burden but greater neutrophil response. ${ }^{66}$ This is important for two reasons: (1) the lower organism burden may decrease the sensitivity of diagnostic tests such as PCR and silver stain ${ }^{66}$ and (2) traditionally employed high-dose corticosteroids may not be effective. ${ }^{67}$

\section{Treatment}

Treatment of fungal infections after lung transplantation is multimodal based on three basic principles. First, antifungal drug therapy should be provided targeting the specific offending pathogen. Second, bronchoscopic and surgical 


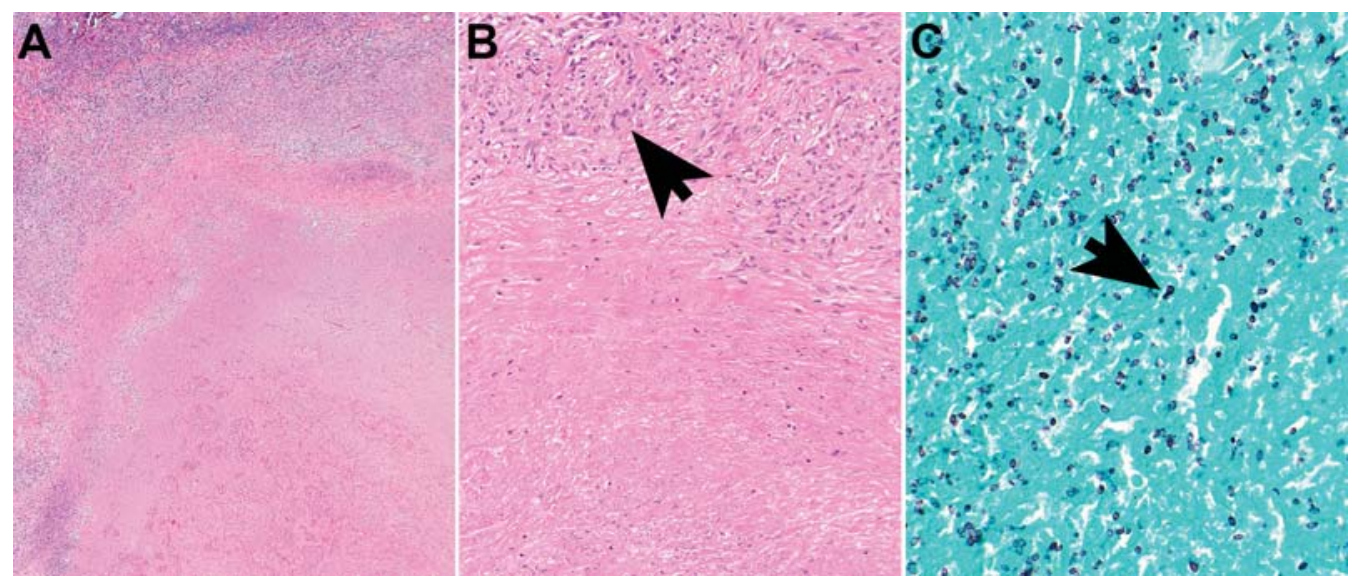

Fig. 6 Necrotizing granuloma with fungal organisms consistent with Histoplasma. (A) Necrotic tissue (right lower side) is lined by a rim of chronic inflammatory cells (left and upper side). (B) Epithelioid histiocytes, a few lymphocytes, and multinucleated giant cells (arrow) rim the necrosis (lower side). (C) A Grocott methenamine silver (GMS) stain highlights small (2-4 $\mu \mathrm{m})$ fungal organisms that show narrow based budding (arrow). Magnification $\times 40$ (A, hematoxylin and eosin), $\times 200$ (B, hematoxylin and eosin), $\times 600$ (C, GMS). (Contributed by Dr. Anja C. Roden, Mayo Clinic Rochester.)

debridement, resection of infected tissue, or removal of infected devices should be performed, as much as feasible, to reduce fungal load. Third, the intensity of immunosuppression should be alleviated to allow for a functional immune system to resolve an opportunistic mycosis.

The choice of antifungal therapy depends on the offending pathogen. In general, antifungal drugs are classified into three different categories: the polyenes, the triazoles, and the echinocandins. - Table $\mathbf{2}$ is a summary of general antifungal treatments for specific fungal infections. Voriconazole is the treatment of choice for invasive aspergillosis, since it has been proven to be more effective than liposomal amphotericin B. Alternative agents for invasive aspergillosis include caspofungin, posaconazole, and isavuconazole. The echinocandins are now the preferred initial empiric treatment of disseminated Candida sp. infections, pending species identification, and antifungal susceptibility testing. High-dose liposomal amphotericin B, up to $10 \mathrm{mg} / \mathrm{kg}$ daily as tolerated, is recommended for the initial empiric treatment of invasive mucormycosis. Liposomal amphotericin B with flucytosine is the initial induction treatment of cryptococcal meningitis, and this is followed by a consolidation and maintenance phase of high-dose fluconazole. Nebulized amphotericin B may help in the treatment of invasive bronchial infections. The antifungal drug terbinafine may be used in selected infections caused by multidrug-resistant fungi.

Therapeutic drug monitoring is recommended for most azole therapies. Suboptimal absorption is a major concern with oral itraconazole. Real-world data with use of SUBAitraconazole (improved bioavailability itraconazole) shows most patients are able to achieve therapeutic trough concentrations at a median of day 7 , thereby circumventing the issues related to standard formulation itraconazole. ${ }^{68}$ In addition, there are ongoing questions of best dosing strategies in areas where there is concern for sub- or supratherapeutic levels, such as obesity, patients in the intensive care units, or those who are receiving extracorporeal membrane oxygenation.
The potential for drug interactions and synergistic toxicities should be considered when using these antifungal agents in lung transplant recipients. The azoles increase the levels of calcineurin inhibitors, such as tacrolimus and cyclosporine, and it is recommended to monitor drug levels of the azoles (to document absorption and prevent toxicities) and the calcineurin inhibitors. Preemptive dose reduction and careful monitoring of serum concentrations of calcineurin inhibitors are needed when azoles are used. Monitoring for renal function is also imperative when an amphotericin B product is prescribed, since it may potentiate the renal toxicities of calcineurin inhibitors. As pharmacologic immunosuppression is reduced to complement the antimicrobial treatment of IFIs, one should be extra cautious to minimize the risk of allograft rejection.

\section{Novel Antifungal Therapies}

An encouraging and promising area of development is the evaluation of the safety and efficacy of novel antifungal therapeutic agents. There are many studies in the early clinical and preclinical phases, with some investigating novel mechanisms of antifungal activity. ${ }^{69}$ These novel compounds are showing promising activity for various IFI, including agents with activity against yeasts, endemic fungi and molds, and resistant fungi.

Fosmanogepix (APX001) is an inhibitor of fungal enzyme Gwt1 with broad anti-yeast, endemic and some mold in vitro activity, and high bioavailability. ${ }^{70}$ It is currently undergoing phase II clinical trials.

Ibrexafungerp is first in class oral triterpenoid inhibitor of $\beta-1,3-D$ glucan synthase inhibitor. It is undergoing phase II and III trials, and promises to be a potential option for oral antifungal therapy with mechanism of action similar to echinocandins. This drug is being evaluated against candida, including Candida auris, and Aspergillus sp.

Olorofim is the first member of a class of antifungals known as orotomides. This class inhibits dihydroorotate dehydrogenase, a key enzyme in the biosynthesis of pyrimidines. It has 
shown broad-spectrum antimold in vitro activity including Scedosporium sp., Lomentospora prolificans, and thermally dimorphic fungi. However, no activity against yeasts or Mucorales sp. is expected. ${ }^{71-73}$ A phase IIB clinical trial is underway for invasive mold infections with limited treatment options (ClinicalTrials.gov identifier: NCT03583164).

Oteseconazole (VT1161) is a tetrazole with an antifungal drug highly specific for CYP51 with expected broad anticandida activity. A phase II trial that compared otesaconazole with fluconazole for vulvovaginitis showed good safety and efficacy results. ${ }^{74}$

Rezafungin is a novel 1,3-beta-D glucan synthase inhibitor with good activity against a broad range of fungal pathogens. A phase II study for invasive candidiasis showed promising results in terms of safety and efficacy. ${ }^{75}$ A phase III clinical trial for candidemia, and another trial evaluating it as a prophylactic agent for Candida spp., Aspergillus spp., and Pneumocystis jirovecii in immunocompromised hosts, is ongoing. ${ }^{76}$ Rezafungin is long-acting and only requires weekly dosing.

\section{Conclusion}

Invasive fungal infections remain important in lung transplant recipients due to immunosuppressed state, impaired natural defenses, and environmental vulnerability. Antifungal prophylaxis is the main strategy for the prevention of IFI following lung transplantation. Although Pneumocystis prophylaxis is continued lifelong, the duration and type of antifungal prophylaxis utilized to prevent most invasive mold infections remains controversial. Furthermore, although some programs practice universal prophylaxis for all recipients, other programs reserve prophylaxis for recipients identified as at increased risk for IFI due to primary diagnosis or pretransplant colonization with fungal organisms. Fungal infections in lung transplant recipients are increasingly diagnosed with the assistance of advanced molecular techniques, which complements (and sometimes circumvents the need for) histopathology. Although common fungal pathogens have not changed considerably, practitioners need to be ever mindful of evolving resistance patterns, as they influence the choices for empiric and targeted therapies. Several new antifungal agents are in the research development pipeline promising improved outcomes.

\section{Funding}

C.C.K. is funded in part by National Institute of Health (NIH) HL 128859. The manuscript's contents are solely the responsibility of the authors and do not necessarily represent the official view of the NIH.

\section{Conflict of Interest}

None declared.

\section{References}

1 Arthurs SK, Eid AJ, Deziel PJ, et al. The impact of invasive fungal diseases on survival after lung transplantation. Clin Transplant 2010;24(03):341-348
2 Bhaskaran A, Mumtaz K, Husain S. Anti-Aspergillus prophylaxis in lung transplantation: a systematic review and meta-analysis. Curr Infect Dis Rep 2013;15(06):514-525

3 Cornely OA, Hoenigl M, Lass-Flörl C, et al; Mycoses Study Group Education and Research Consortium (MSG-ERC) and the European Confederation of Medical Mycology (ECMM) Defining breakthrough invasive fungal infection-position paper of the mycoses study group education and research consortium and the European Confederation of Medical Mycology. Mycoses 2019;62(09):716-729

4 Doligalski CT, Benedict K, Cleveland AA, et al. Epidemiology of invasive mold infections in lung transplant recipients. Am J Transplant 2014;14(06):1328-1333

5 Vazquez R, Vazquez-Guillamet MC, Suarez J, Mooney J, Montoya JG, Dhillon GS. Invasive mold infections in lung and heart-lung transplant recipients: Stanford University experience. Transpl Infect Dis 2015;17(02):259-266

6 Ashraf N, Kubat RC, Poplin V, et al. Re-drawing the maps for endemic mycoses. Mycopathologia 2020;185(05):843-865

7 Maphanga TG, Birkhead M, Muñoz JF, et al. Human blastomycosis in South Africa caused by Blastomyces percursus and Blastomyces emzantsi sp. nov., 1967 to 2014. J Clin Microbiol 2020;58(03):58

8 Oltean HN, Springer M, Bowers JR, et al. Suspected locally acquired coccidioidomycosis in human, Spokane, Washington, USA. Emerg Infect Dis 2020;26(03):606-609

9 Lestrade PPA, Buil JB, van der Beek MT, et al. Paradoxal trends in azole-resistant Aspergillus fumigatus in a national multicenter surveillance program, the Netherlands, 2013-2018. Emerg Infect Dis 2020;26(07):1447-1455

10 Zhang J, Lopez Jimenez L, Snelders E, et al. Dynamics of Aspergillus fumigatus in azole fungicide-containing plant waste in the Netherlands (2016-2017). Appl Environ Microbiol 2021;87(02):e02295-e20

11 Baddley JW, Marr KA, Andes DR, et al. Patterns of susceptibility of Aspergillus isolates recovered from patients enrolled in the Transplant-Associated Infection Surveillance Network. J Clin Microbiol 2009;47(10):3271-3275

12 Delbove A, Alami H, Tissot A, et al. Pneumocystis pneumonia after lung transplantation: a retrospective multicenter study. Respir Med 2020;169:106019

13 Neoh CF, Snell GI, Kotsimbos T, et al. Antifungal prophylaxis in lung transplantation-a world-wide survey. Am J Transplant 2011; 11(02):361-366

14 Pennington KM, Yost KJ, Escalante P, Razonable RR, Kennedy CC. Antifungal prophylaxis in lung transplant: a survey of United States' transplant centers. Clin Transplant 2019;33(07):e13630

15 Pennington KM, Baqir M, Erwin PJ, Razonable RR, Murad MH, Kennedy CC. Antifungal prophylaxis in lung transplant recipients: a systematic review and meta-analysis. Transpl Infect Dis 2020;22 (04):e13333

16 Pennington KM, Razonable RR, Peters S, et al. Why do lung transplant patients discontinue triazole prophylaxis? Transpl Infect Dis 2019;21(03):e13067

17 Pennington KM, Dykhoff HJ, Yao X, et al. The impact of antifungal prophylaxis in lung transplant recipients. Ann Am Thorac Soc 2021;18(03):468-476

18 Neoh CF, Snell GI, Levvey B, et al. Preemptive treatment with voriconazole in lung transplant recipients. Transpl Infect Dis 2013;15(04):344-353

19 Pilarczyk K, Haake N, Heckmann J, et al. Is universal antifungal prophylaxis mandatory in adults after lung transplantation? A review and meta-analysis of observational studies. Clin Transplant 2016;30(12):1522-1531

20 Lass-Flörl C, Aigner M, Nachbaur D, et al. Diagnosing filamentous fungal infections in immunocompromised patients applying computed tomography-guided percutaneous lung biopsies: a 12-year experience. Infection 2017;45(06):867-875

21 Bart-Delabesse E, Basile M, Al Jijakli A, et al. Detection of Aspergillus galactomannan antigenemia to determine biological and 
clinical implications of beta-lactam treatments. J Clin Microbiol 2005;43(10):5214-5220

22 Castagnola E, Machetti M, Bucci B, Viscoli C. Antifungal prophylaxis with azole derivatives. Clin Microbiol Infect 2004;10 (Suppl 1):86-95

23 Pfeiffer CD, Fine JP, Safdar N. Diagnosis of invasive aspergillosis using a galactomannan assay: a meta-analysis. Clin Infect Dis 2006;42(10):1417-1427

24 Park SY, Lee SO, Choi SH, et al. Aspergillus galactomannan antigen assay in bronchoalveolar lavage fluid for diagnosis of invasive pulmonary aspergillosis. J Infect 2010;61(06):492-498

25 Bergeron A, Belle A, Sulahian A, et al. Contribution of galactomannan antigen detection in BAL to the diagnosis of invasive pulmonary aspergillosis in patients with hematologic malignancies. Chest 2010;137(02):410-415

26 Guo YL, Chen YQ Wang K, Qin SM, Wu C, Kong JL. Accuracy of BAL galactomannan in diagnosing invasive aspergillosis: a bivariate metaanalysis and systematic review. Chest 2010;138(04): 817-824

27 Heng SC, Morrissey O, Chen SC, et al. Utility of bronchoalveolar lavage fluid galactomannan alone or in combination with PCR for the diagnosis of invasive aspergillosis in adult hematology patients: a systematic review and meta-analysis. Crit Rev Microbiol 2015;41(01):124-134

28 Luong ML, Filion C, Labbé AC, et al. Clinical utility and prognostic value of bronchoalveolar lavage galactomannan in patients with hematologic malignancies. Diagn Microbiol Infect Dis 2010;68 (02):132-139

29 Racil Z, Kocmanova I, Toskova M, et al. Galactomannan detection in bronchoalveolar lavage fluid for the diagnosis of invasive aspergillosis in patients with hematological diseases-the role of factors affecting assay performance. Int J Infect Dis 2011;15(12): e874-e881

30 Nucci M, Carlesse F, Cappellano P, et al. Earlier diagnosis of invasive fusariosis with Aspergillus serum galactomannan testing. PLoS One 2014;9(01):e87784

31 Busca A, Locatelli F, Barbui A, et al. Usefulness of sequential Aspergillus galactomannan antigen detection combined with early radiologic evaluation for diagnosis of invasive pulmonary aspergillosis in patients undergoing allogeneic stem cell transplantation. Transplant Proc 2006;38(05):1610-1613

32 Obayashi T, Yoshida M, Mori T, et al. Plasma (1->3)-beta-Dglucan measurement in diagnosis of invasive deep mycosis and fungal febrile episodes. Lancet 1995;345(8941):17-20

33 Hage CA, Carmona EM, Evans SE, Limper AH, Ruminjo J, Thomson CC. Summary for clinicians: microbiological laboratory testing in the diagnosis of fungal infections in pulmonary and critical care practice. Ann Am Thorac Soc 2019;16(12):1473-1477

34 Bansal N, Gopalakrishnan R, Sethuraman N, et al. Experience with $\beta$-D-glucan assay in the management of critically ill patients with high risk of invasive candidiasis: an observational study. Indian J Crit Care Med 2018;22(05):364-368

35 Posteraro B, Tumbarello M, De Pascale G, et al. (1,3)- $\beta$-D-glucanbased antifungal treatment in critically ill adults at high risk of candidaemia: an observational study. J Antimicrob Chemother 2016;71(08):2262-2269

36 Tran T, Beal SG. Application of the 1,3- $\beta$-D-glucan (Fungitell) assay in the diagnosis of invasive fungal infections. Arch Pathol Lab Med 2016;140(02):181-185

37 Wood BR, Komarow L, Zolopa AR, Finkelman MA, Powderly WG, Sax PE. Test performance of blood beta-glucan for Pneumocystis jirovecii pneumonia in patients with AIDS and respiratory symptoms. AIDS 2013;27(06):967-972

38 Cruciani M, Mengoli C, Loeffler J, et al. Polymerase chain reaction blood tests for the diagnosis of invasive aspergillosis in immunocompromised people. Cochrane Database Syst Rev 2015: CD009551
39 Springer J, Goldenberger D, Schmidt F, et al. Development and application of two independent real-time PCR assays to detect clinically relevant Mucorales species. J Med Microbiol 2016;65 (03):227-234

40 Avni T, Levy I, Sprecher H, Yahav D, Leibovici L, Paul M. Diagnostic accuracy of PCR alone compared to galactomannan in bronchoalveolar lavage fluid for diagnosis of invasive pulmonary aspergillosis: a systematic review. J Clin Microbiol 2012;50(11):3652-3658

41 Neely LA, Audeh M, Phung NA, et al. T2 magnetic resonance enables nanoparticle-mediated rapid detection of candidemia in whole blood. Sci Transl Med 2013;5(182):182ra54

42 Mylonakis E, Clancy CJ, Ostrosky-Zeichner L, et al. T2 magnetic resonance assay for the rapid diagnosis of candidemia in whole blood: a clinical trial. Clin Infect Dis 2015;60(06):892-899

43 Clancy CJ, Nguyen MH. Diagnosing invasive candidiasis. J Clin Microbiol 2018;56(05):e01909-e01917

44 Walker B, Powers-Fletcher MV, Schmidt RL, Hanson KE. Costeffectiveness analysis of multiplex PCR with magnetic resonance detection versus empiric or blood culture-directed therapy for management of suspected candidemia. J Clin Microbiol 2016;54 (03):718-726

45 Durkin M, Witt J, Lemonte A, Wheat B, Connolly P. Antigen assay with the potential to aid in diagnosis of blastomycosis. J Clin Microbiol 2004;42(10):4873-4875

46 Bariola JR, Hage CA, Durkin M, et al. Detection of Blastomyces dermatitidis antigen in patients with newly diagnosed blastomycosis. Diagn Microbiol Infect Dis 2011;69(02):187-191

47 Frost HM, Novicki TJ. Blastomyces antigen detection for diagnosis and management of blastomycosis. J Clin Microbiol 2015;53(11): 3660-3662

48 Fandiño-Devia E, Rodríguez-Echeverri C, Cardona-Arias J, Gonzalez A. Antigen Detection in the Diagnosis of Histoplasmosis: A Meta-analysis of Diagnostic Performance. Mycopathologia 2016; 181(3-4):197-205

49 Richer SM, Smedema ML, Durkin MM, et al. Improved diagnosis of acute pulmonary histoplasmosis by combining antigen and antibody detection. Clin Infect Dis 2016;62(07):896-902

50 Klein BS, Vergeront JM, Weeks RJ, et al. Isolation of Blastomyces dermatitidis in soil associated with a large outbreak of blastomycosis in Wisconsin. N Engl J Med 1986;314(09):529-534

51 Richer SM, Smedema ML, Durkin MM, et al. Development of a highly sensitive and specific blastomycosis antibody enzyme immunoassay using Blastomyces dermatitidis surface protein BAD-1. Clin Vaccine Immunol 2014;21(02):143-146

52 Malo J, Holbrook E, Zangeneh T, et al. Enhanced antibody detection and diagnosis of coccidioidomycosis with the Miravista IgG and IgM detection enzyme immunoassay. J Clin Microbiol 2017; 55(03):893-901

53 Lortholary O, Renaudat C, Sitbon K, et al; French Mycosis Study Group. Worrisome trends in incidence and mortality of candidemia in intensive care units (Paris area, 2002-2010). Intensive Care Med 2014;40(09):1303-1312

54 Andes DR, Safdar N, Baddley JW, et al; Mycoses Study Group. Impact of treatment strategy on outcomes in patients with candidemia and other forms of invasive candidiasis: a patientlevel quantitative review of randomized trials. Clin Infect Dis 2012;54(08):1110-1122

55 Chamilos G, Luna M, Lewis RE, et al. Invasive fungal infections in patients with hematologic malignancies in a tertiary care cancer center: an autopsy study over a 15-year period (1989-2003). Haematologica 2006;91(07):986-989

56 Carneiro HA, Coleman JJ, Restrepo A, Mylonakis E. Fusarium infection in lung transplant patients: report of 6 cases and review of the literature. Medicine (Baltimore) 2011;90(01):69-80

57 Roden MM, Zaoutis TE, Buchanan WL, et al. Epidemiology and outcome of zygomycosis: a review of 929 reported cases. Clin Infect Dis 2005;41(05):634-653 
58 Stewart JI, D’Alonzo GE, Ciccolella DE, Patel NB, Durra H, Clauss HE. Reverse halo sign on chest imaging in a renal transplant recipient. Transpl Infect Dis 2014;16(01):115-118

59 Wand O, Unterman A, Izhakian S, Fridel L, Kramer MR. Mucormycosis in lung transplant recipients: a systematic review of the literature and a case series. Clin Transplant 2020;34(02):e13774

60 Marek C, Croxen MA, Dingle TC, et al. The use of genome sequencing to investigate an outbreak of hospital-acquired mucormycosis in transplant patients. Transpl Infect Dis 2019; 21(05):e13163

61 Chaves MS, Franco D, Nanni JC, et al. Control of an outbreak of postoperative bone mucormycosis: an intervention study of contiguous cohorts. Am J Infect Control 2016;44(12):1715-1717

62 Walther G, Wagner L, Kurzai O. Outbreaks of Mucorales and the species involved. Mycopathologia 2020;185(05):765-781

63 Limper AH, Knox KS, Sarosi GA, et al; American Thoracic Society Fungal Working Group. An official American Thoracic Society statement: treatment of fungal infections in adult pulmonary and critical care patients. Am J Respir Crit Care Med 2011;183(01):96-128

64 Miller R, Assi MAST Infectious Diseases Community of Practice. Endemic fungal infections in solid organ transplant recipientsGuidelines from the American Society of Transplantation Infectious Diseases Community of Practice. Clin Transplant 2019;33 (09):e13553

65 Kauffman CA, Freifeld AG, Andes DR, et al. Endemic fungal infections in solid organ and hematopoietic cell transplant recipients enrolled in the Transplant-Associated Infection Surveillance Network (TRANSNET). Transpl Infect Dis 2014;16(02):213-224

66 Thomas CF Jr, Limper AH. Pneumocystis pneumonia. N Engl J Med 2004;350(24):2487-2498

67 Wieruszewski PM, Barreto EF, Barreto JN, et al. Preadmission corticosteroid therapy and the risk of respiratory failure in adults without HIV presenting with Pneumocystis pneumonia. J Intensive Care Med 2020;35(12):1465-1470
68 Nield B, Larsen SR, van Hal SJ. Clinical experience with new formulation $S U B A \circledast$-itraconazole for prophylaxis in patients undergoing stem cell transplantation or treatment for haematological malignancies. J Antimicrob Chemother 2019;74(10):3049-3055

69 Johnson MD, Lewis RE, Dodds Ashley ES, et al. Core recommendations for antifungal stewardship: a statement of the Mycoses Study Group Education and Research Consortium. J Infect Dis 2020;222(Suppl 3):S175-S198

70 Shaw KJ, Ibrahim AS. Fosmanogepix: a review of the first-in-class broad spectrum agent for the treatment of invasive fungal infections. J Fungi (Basel) 2020;6(04):6

71 Astvad KMT, Jørgensen KM, Hare RK, Datcu R, Arendrup MC. Olorofim susceptibility testing of 1,423 Danish mold isolates obtained in 2018-2019 confirms uniform and broad-spectrum activity. Antimicrob Agents Chemother 2020;65(01):65

72 Rivero-Menendez O, Cuenca-Estrella M, Alastruey-Izquierdo A. In vitro activity of olorofim against clinical isolates of Scedosporium species and Lomentospora prolificans using EUCAST and CLSI methodologies. J Antimicrob Chemother 2020;75(12):3582-3585

73 Singh A, Singh P, Meis JF, Chowdhary A. In vitro activity of the novel antifungal olorofim against dermatophytes and opportunistic moulds including Penicillium and Talaromyces species. J Antimicrob Chemother 2021:dkaa562

74 Brand SR, Sobel JD, Nyirjesy P, et al. Randomized phase 2 study of VT-1161 for the treatment of acute vulvovaginal candidiasis. Clin Infect Dis 2020:ciaa1204. doi: 10.1093/cid/ciaa1204

75 Thompson GR, Soriano A, Skoutelis A, et al. Rezafungin versus caspofungin in a phase 2 , randomized, double-blind study for the treatment of candidemia and invasive candidiasis-The STRIVE trial. Clin Infect Dis 2020:ciaa1380. doi: 10.1093/cid/ciaa1380

76 Miesel L, Cushion MT, Ashbaugh A, Lopez SR, Ong V. Efficacy of rezafungin in prophylactic mouse models of invasive Candidiasis, Aspergillosis, and Pneumocystis pneumonia. Antimicrob Agents Chemother 2021;65(03):e01992-20 